\title{
ISM turbulence driven by the magnetorotational instability
}

\author{
Robert A. Piontek ${ }^{1,2}$ and Eve C. Ostriker ${ }^{2}$ \\ ${ }^{1}$ Astrophysikalisches Institut Potsdam, An der Sternwarte 16, D-14482 Potsdam, Germany \\ email: rpiontek@aip.de \\ ${ }^{2}$ Department of Astronomy, University of Maryland, College Park, MD 20742-2421 \\ email: ostriker@astro.umd.edu
}

\begin{abstract}
We have performed numerical simulations which were designed to further our understanding of the turbulent interstellar medium (ISM). Our simulations include a multi-phase thermodynamic model of the ISM, magnetic fields, and sheared rotation, allowing us to study the effects of the magnetorotational instability (MRI) in an environment containing high density cold clouds embedded in a warm, low density, ambient medium. These models have shown that the MRI is indeed a significant source of turbulence, particularly at low mean densities typical of the outer regions of the Milky Way, where star formation rates are low, but high levels of turbulence persist. Here, we summarize past findings, as well as our most recent models which include vertical stratification, allowing us to self-consistently model the vertical distribution of material in the disk.
\end{abstract}

Keywords. ISM: general, clouds, kinematics and dynamics, magnetic fields, structure

\section{Introduction}

In the traditional picture of the ISM (Cox \& Smith 1974; McKee \& Ostriker 1977; Spitzer 1978) turbulence is driven primarily by supernova explosions. In more recent years, however, some observations of the Milky Way and external galaxies have lead us to believe that another significant source of turbulence must be at work in the ISM. In short, the observed velocity dispersion of neutral hydrogen is found to be relatively constant, while the SNe rate is not. In particular, turbulent velocities of neutral hydrogen are high in the outer regions of galaxies (Dickey et al. 1990), and arm/inter-arm regions show no difference in the measured velocity dispersion (Dickey et al. 1990). If turbulence is driven by $\mathrm{SNe}$, we would expect that turbulent velocities should be larger near regions of active star formation. These observations motivated Sellwood \& Balbus (1999) to propose that turbulence in the ISM may be driven by the magnetorotational instability, and over the past few years we have investigated this theory with numerical simulations.

The MRI has been extensively studied in the context of accretion disks (Hawley \& Balbus 1992; Stone et al. 1996). The ISM meets the basic requirements to be unstable to the MRI: it is a rotating-shearing system, with a weak magnetic field. However, it is different from typical accretion disk systems in one important aspect. The ISM is structured by radiative heating and cooling processes (Wolfire et al. 2003) so that it is found in two distinct "phases". A high density, clumpy, cold phase is thought to be embedded in a warm, diffuse, low density intercloud medium (Field et al. 1969). Gas at intermediate temperatures is thermally unstable (Field 1965), and heats or cools to join either the warm or cold stable phases. Our aim has been to study the MRI in the presence of a two-phase medium. Here we briefly summarize results from Piontek \& 


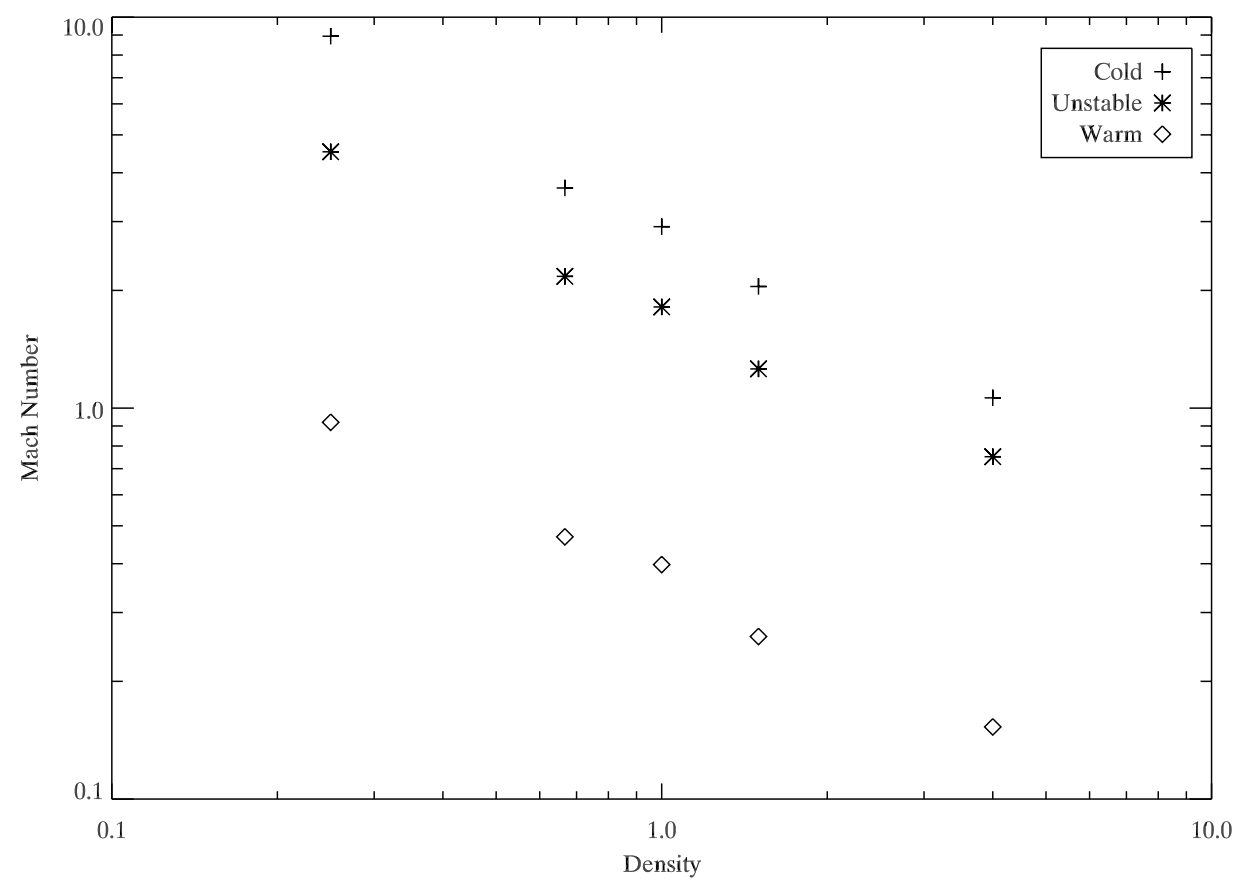

Figure 1. For five different simulations with varying mean density, we plot the time averaged, mass weighted, turbulent Mach number, for the warm, unstable, and cold phases of gas. Our lowest density model has a turbulent velocity of $8 \mathrm{~km} \mathrm{~s}^{-1}$.

Ostriker (2004), Piontek \& Ostriker (2005), and new, unpublished results from a recently submitted paper to $A p J$.

\section{Simulations and results}

We performed MHD simulations using an MPI parallel version of ZEUS (Stone \& Norman 1992a, 1992b), that includes a radiative cooling function adopted from SánchezSalcedo et al. (2002), which allows us to simulate a multi-phase ISM. As previously described, there are two stable phases of gas, a cold (100 K), high density, clumpy phase, and a warm $(8000 \mathrm{~K})$, low density, intercloud medium. Without a turbulent source, very little gas exists at intermediate temperatures, but turbulence can force gas into the unstable regime. Our simulations are performed in the local, rotating reference frame of the galaxy, and our simulation domain represents a small patch of the ISM, measuring 100-300 pc on each side, depending on the model. The initial magnetic field strength is $0.26 \mu G$, and is vertical. We include shear from the galactic rotation curve using shearing-periodic boundary conditions (Hawley \& Balbus 1992; Hawley et al. 1995), which in combination with magnetic fields, results in MRI driven turbulence. For details concerning the numerical method, please see Piontek \& Ostriker (2004). The primary question we wish to address is whether or not the MRI can produce turbulence at the level which is observed in the Milky Way and other galaxies, which is typically around $7 \mathrm{~km} \mathrm{~s}^{-1}$ Heiles \& Troland (2003).

In Piontek \& Ostriker (2005), we performed five different simulations, varying only the mean density, from $\bar{n}=0.25 \mathrm{~cm}^{-3}$ to $\bar{n}=4 \mathrm{~cm}^{-3}$. We found that the combination of a 


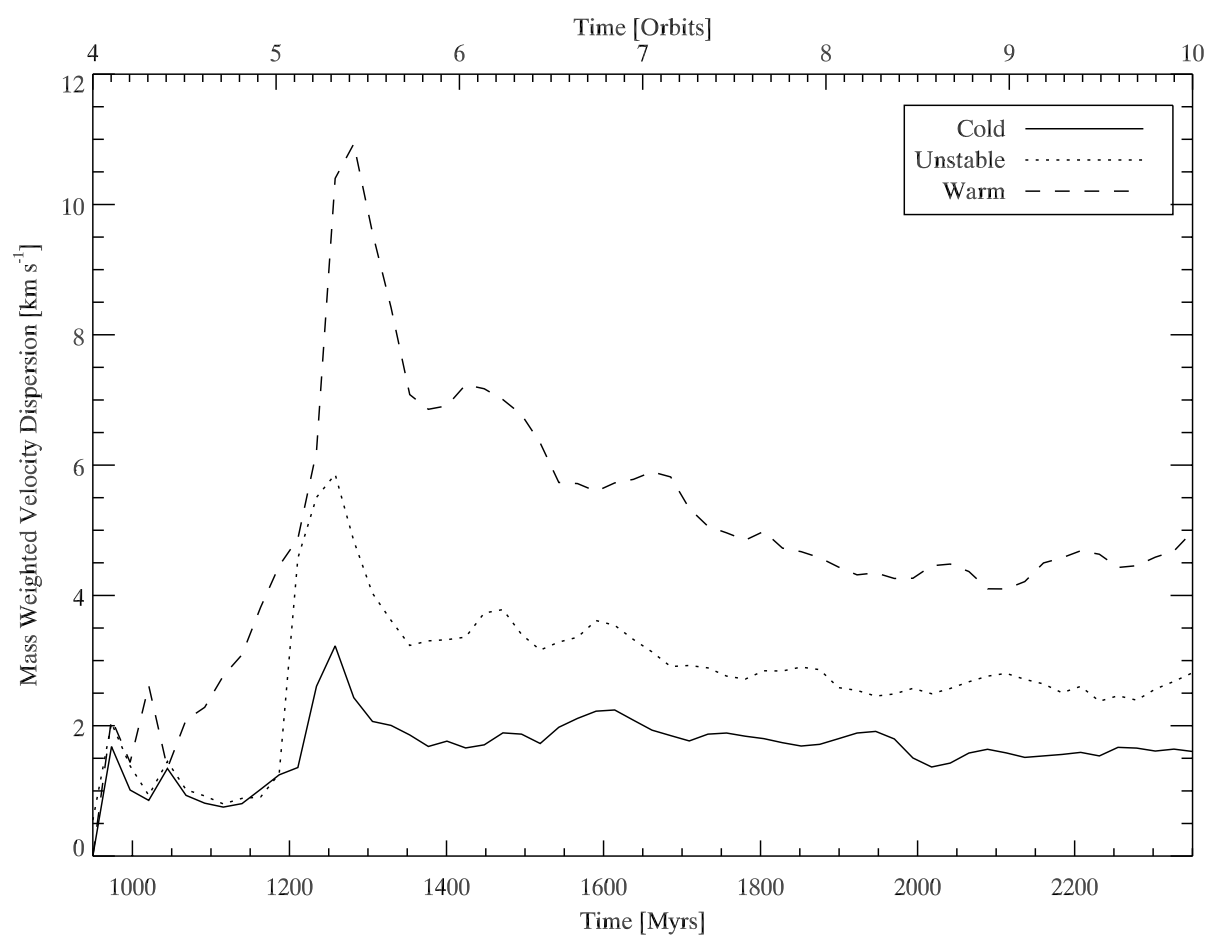

Figure 2. Mass weighted velocity dispersion as a function of time for our inner galaxy stratified model. It can be seen here that the cold component has a much lower velocity dispersion compared to the warm component. This is because the cold component sinks to the mid-plane, increasing the local mean density. In our unstratified models, all three phases were driven at approximately the same velocity.

multi-phase ISM with the MRI results in a scaling of turbulent velocity with mean density that is steeper than what is expected for the MRI in a single-phase model of ISM. As the mean density decreases, MRI turbulence grows more quickly in a multi-phase ISM than it otherwise would. In figure 1 we plot the time averaged, mass weighted, turbulent Mach number as a function of mean density for five different simulations, with each thermal component shown individually. All three components are driven at approximately the same velocity at any particular mean density, but since each phase has a fairly well defined temperature associated with it, the Mach numbers vary significantly. The warm phase is subsonic, and the cold phase is supersonic, and in our model with the lowest mean density, $\bar{n}=0.25 \mathrm{~cm}^{-3}$, these Mach numbers are about 0.15 , and 9.0 , respectively. This corresponds to a velocity of approximately $8 \mathrm{~km} \mathrm{~s}^{-1}$. This result lead us to conclude that the MRI may indeed be an important source of turbulence in low density environments such as the outer regions of the Milky Way.

We have recently performed simulations which additionally include vertical stratification. In these models the local mean density is determined self consistently, whereas previously the mean density was simply a parameter (though the total mass in the model still remains a parameter). Our simulation domain for these stratified models extends 900 pc above and below the mid-plane. In these models we find that the higher density cold clouds tend to sink to the mid-plane. Here they shield themselves from the more turbulent warm medium, and the turbulent velocity of the cold component is lower than what we find for our non-stratified models. 
In figure 2 we plot the mass weighted velocity dispersion as a function of time for our standard stratified model, with a surface density of $\Sigma=10 M_{\odot} \mathrm{pc}^{-2}$. The warm medium saturates at around $4-5 \mathrm{~km} / \mathrm{s}$, approximately the level which is observed, but the cold medium saturates at $1-2 \mathrm{~km} / \mathrm{s}$, which is too small to account for observed velocity dispersions. The cold, high density clouds sink to the mid-plane, and the local mean density increases. The increased mean density causes the MRI to be less effective at driving turbulence, as we found in our unstratified models. The MRI is unable to lift the cold gas off of the mid-plane, so that the scale heights we measure in our stratified disks are too small compared to observations. We also performed models of the outer disk with a lower surface density, $\Sigma=6 M_{\odot} \mathrm{pc}^{-2}$. This model has a cold mass fraction of only $16 \%$, with a scale height of around $150 \mathrm{pc}$. In this case the velocity dispersions increase to $6.6,3.3$, and $2.5 \mathrm{~km} / \mathrm{s}$ for the warm, unstable, and cold phases. We also find that the turbulent magnetic pressure is approximately twice the thermal pressure for both our inner and outer galaxy models, which is consistent with observations.

\section{Conclusions}

Our unstratified simulations have shown that the MRI may indeed be an important source of turbulence in the ISM, especially when the mean density is low, as is true in the outer galaxy. Our new stratified models, however, have cold component turbulent velocities which are too small compared to observations. A much more detailed analysis of these stratified models will be presented in a forthcoming paper.

\section{Acknowledgements}

This work was supported in part by grants NAG5-9167 (NASA), AST 0205972 and AST 0507315 (National Science Foundation). Most of the simulations presented here were performed on the Thunderhead cluster at Goddard Space Flight Center, and the Center for Theory and Computation cluster in the University of Maryland Department of Astronomy. This research has made use of NASA's Astrophysics Data System.

\section{References}

Cox, D. P. \& Smith, B. W. 1974, ApJ 189, L105

Dickey, J. M., Hanson, M. M. \& Helou, G. 1990, ApJ 352, 522

Field, G. B., Goldsmith, D. W. \& Habing, H. J. 1969, ApJL 155, L149

Field, G. B. 1965, ApJ 142, 531

Hawley, J. F. \& Balbus, S. A. 1992, ApJ 400, 595

Hawley, J. F., Gammie, C. F. \& Balbus, S. A. 1995, ApJ 440, 742

Heiles, C. \& Troland, T. H. 2003, ApJ 586, 1067

McKee, C. F. \& Ostriker, J. P. 1977, ApJ 218, 148

Piontek, R. A. \& Ostriker, E. C. 2004, ApJ 601, 905

Piontek, R. A. \& Ostriker, E. C. 2005, ApJ 629, 849

Sánchez-Salcedo, F. J., Vázquez-Semadeni, E. \& Gazol, A. 2002, ApJ 577, 768

Sellwood, J. A. \& Balbus, S. A. 1999, ApJ 511, 660

Spitzer, L. 1978, Physical processes in the interstellar medium (New York Wiley-Interscience), p. 333

Stone, J. M. \& Norman, M. L. 1992a, ApJS 80, 753

Stone, J. M. \& Norman, M. L. 1992b, ApJS 80, 791

Stone, J. M., Hawley, J. F., Gammie, C. F. \& Balbus, S. A. 1996, ApJ 463, 656

Wolfire, M. G., McKee, C. F., Hollenbach, D. \& Tielens, A. G. G. M. 2003, ApJ 587, 278 


\section{Discussion}

OmukAi: (1.) Does your simulation satisfy the so-called Field condition? Namely, if the simulation fails to resolve the Field length, it is know that artificial structures are created. (2.) The conduction depends on the magnetic field orientation. Did you include this effect?

PIONTEK: (1.) Yes, we include thermal conduction at a level which allows us to resolve the most unstable mode of the thermal instability. (2.) This is not accounted for. The level of conduction is constant throughout the simulation domain.

Krumholz: Can you suggest observational diagnostic to distinguish supernova-driven turbulence from MRI-driven turbulence?

PIONTEK: MRI driven turbulence is stronger in the radial direction than the vertical. It is possible that this could be observed.

CRUtcher: You get magnetic field strengths of about $3 \mu \mathrm{G}$, yet the observed strengths are twice that in the Milky Way, and twice again stronger in some galaxies. Would a stronger initial field still have allowed your MRI to work?

PIONTEK: The saturation of the magnetic field strength is determined by the thermal pressure, which is set by the cooling function we've used, and the thermal pressure is somewhat low in our model. Higher thermal pressures would give higher magnetic field strengths. Very strong fields, however, will stabilize the MRI. 